
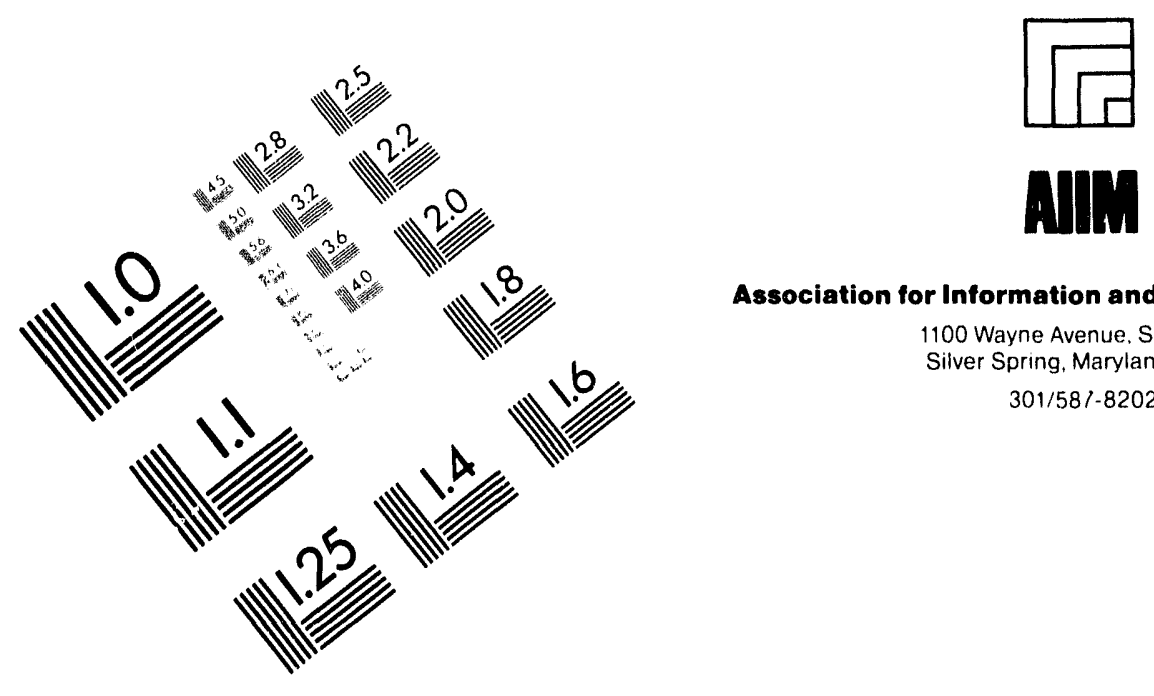

Association for Information and Image Management

1100 Wayne Avenue. Suite 1100

Silver Spring, Maryland 20910

301/58/-8202

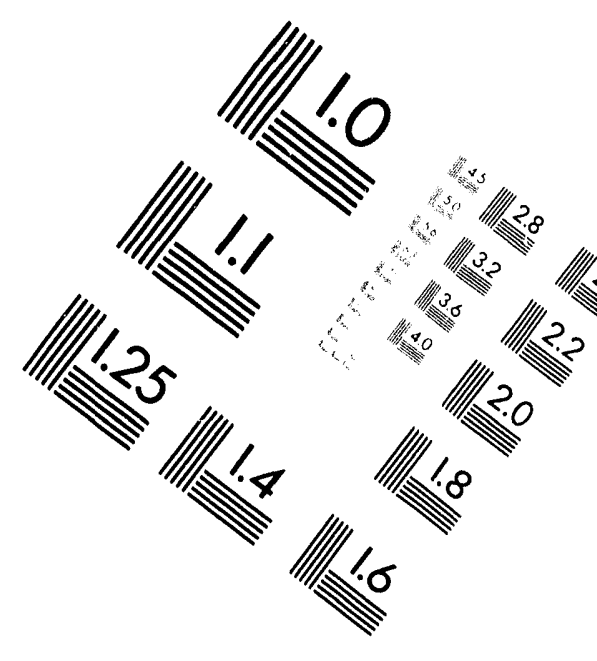

\title{
Centimeter
}

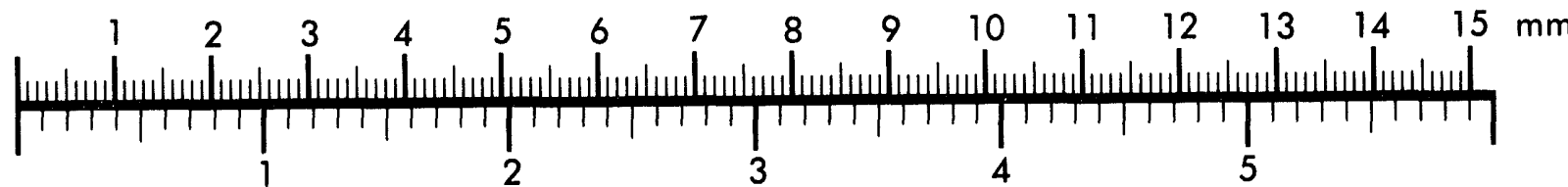

Inches
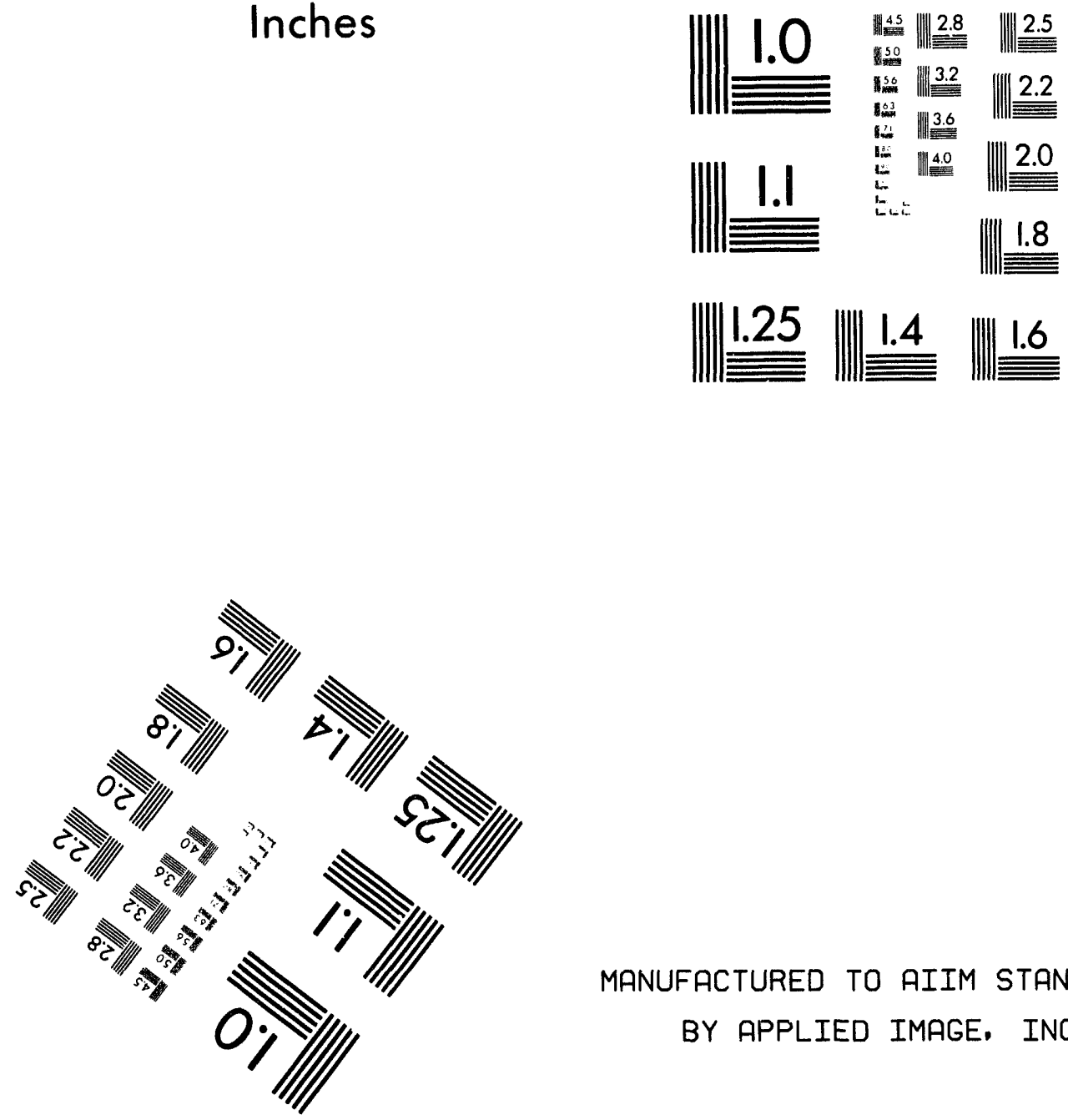

MANUFACTURED TO AIIM STANDARDS

BY APPLIED IMAGE. INC.

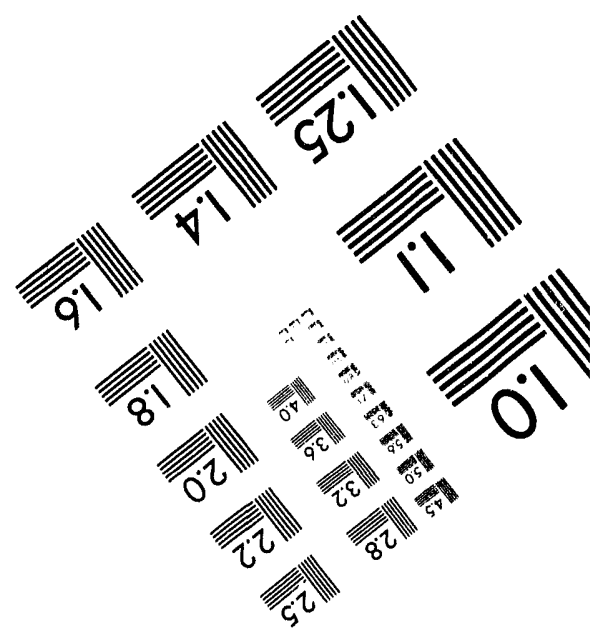



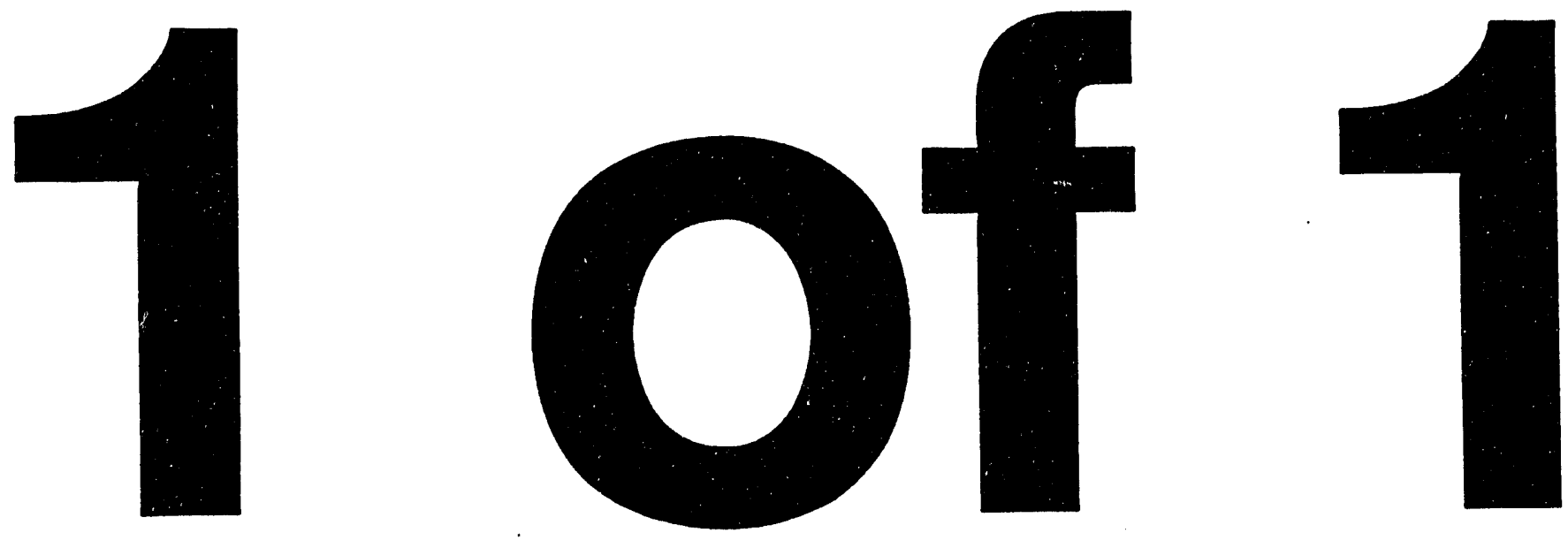


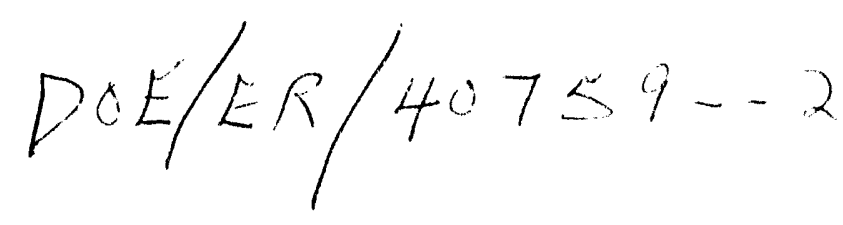

\section{THEORETICAL PROBLEMS IN ACCELERATOR PHYSICS}

\section{PROGRESS REPORT \\ DE-FG03-93ER40759}

This is the second progress report submitted under my current grant and covers progress made since the submission of the first progress report in August 1993. During this period I have continued to spend approximately one half of my time at SLAC and most of the projects reported here were carried out in collaboration with individuals and groups at SLAC. Except where otherwise noted, reference numbers in the text refer to the attached list of current contract publications. Copies of the publications, numbered in agreement with the publication list, are included with this report.

\section{ACCELERATOR STRUCTURE DESIGN AND SIMULATION}

The current status and history of the SLAC program for the design and simulation of accelerating structures for future linear colliders is described in ref [1], a long paper which was completed during the past year and is scheduled to appear in Particle Accelerators. The central issue discussed in this paper is the suppression of transverse wakefields. Two methods are discussed: (1) Waveguide damping of individual cells. Here work which has been reported in a number of our previous progress reports and in the final report of our previous contract is summarized and (2) detuning, i.e. varying the frequencies of the higher-order modes (HOM's) so that the net wakefield induced by the beam rapidly decoheres. This latter approach has been the basis of the design of the cavities for the NLC test accelerator (NLCTA). These 1.8 meter cavities are disc loaded structures consisting of 204 cells plus two couplers. The design principles, theory, and predicted transverse wakefields are given in ref [1]. Very recently, measurements in the ASSET experiment have confirmed the predicted wakefield suppression. Due mainly to the discreteness but also the finite extent of the spectra of the detuned HOM's, at distances of about 4 meters behind the generating bunch the wakefield begins to increase again and becomes unacceptably large for s greater than 10 meters or so, thus limiting the number of bunches which can be accelerated without excessive emittance growth. The solution to this problem suggested in [1] is to reduce the effective mode spacing by interleaving the detuned frequency spectrum of four successive 1.8 meter sections. This procedure is shown to be very effective provided uncomfortably strict tolerances can be maintained.

An alternative solution, which has been apparent all along, would be to suppress the long range wakefield by providing weak damping as a supplement to the detuning. The issue has always been whether or not weak damping was any easier to supply than strong damping. With the latter, of course, the detuning becomes superfluous. The idea that such damping might be provided by coupling all cavities to several identical and symmetrically located waveguides (manifolds) which run parallel to the accelerator structure and are terminated at each end by matched loads has, as mentioned in [1], been under consideration for some time. We report here two critical new points which have led to a breakthrough in our understanding of how such a scheme might work:

(1) One can couple the accelerator cavities directly to the manifolds through large coupling irises without significantly damping the accelerating mode (AM). That is, a frequency discriminating assembly to protect the AM for each cavity, which is required when the cavities are damped individually, is not needed (These have typically been included in heavy damping schemes such as the circumferential slot damping discussed

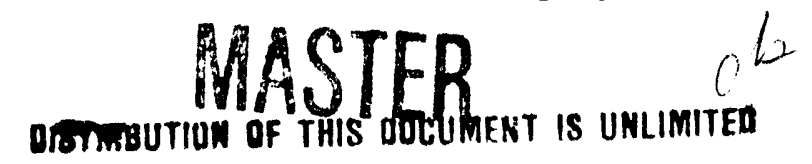


in [1] and also the Shintake choke mode cavity). The discrimination can be provided instead via the mode structure of the manifolds and also via the mismatch of phase velocity between the $\mathrm{AM}$ and whatever manifold modes propagate at the accelerator frequency.

(2) The HOM's excited by the particle beam are not traveling waves propagating at the beam velocity but are, due to HOM detuning, broadly localized standing waves, each of which has a broad spectrum of phase velocities [6]. These may therefore couple reasonably well to the more numerous waveguide modes which propagate at the HOM frequencies.

With reference to point (1) further consideration has led us to opt for discrimination based upon waveguide cutoff considerations. The phase velocity mismatch effect is very effective during steady state, but is less effective during pulse build up and decay. While this may not be serious from the point of view of efficiency, it could lead to a larger than necessary power deposition in the loads. As discussed in [6] the manifolds have been designed so that they provide no damping for the accelerating mode. All waveguide modes are cutoff at the accelerating mode frequency except the lowest, and its symmetry is such that it is totally decoupled from the accelerating mode. There is some reduction in shunt impedance of the accelerating mode associated with coupling to the manifold, and a key current design problem is determining a proper balance between this undesirable effect and the damping which can be obtained by tight coupling.

Reference [6] is the draft of an extended version of a paper to be included in the proceedings of LINAC94 (Tsukuba). (A paper on the same subject may also appear in the Advanced Accelerator Concepts Conference Proceedings (Fontana, Wi), as a brief presentation of this work was made there.) It provides a description of the theoretical work which has so far been carried out to explore this approach in a more quantitative way. While it has led us to conclude that the approach is very promising, there are critical design considerations of the sort mentioned in the previous paragraph which urgently need a stronger theoretical basis. Of particular importance is the inclusion of the full spectrum of propagating manifold modes and the improved theory of cell-manifold coupling which we are in the process of developing.

One undamped detuned 1.8 meter section for the NLCTA has been constructed and a second is in process. Serious consideration involving mechanical design, engineering, and scheduling is being given to including this damping scheme in the third and fourth. The theoretical problem of understanding this damping scheme is sufficiently complex to make a parallel experimental investigation very important. We note that in contrast to the undamped detuned structure a great deal of information can be obtained via cold testing. This is because the ends of the manifolds provide access to the modes which are localized within the structure, and the transmission of RF through the cells from one manifold to another can be measured. Furthermore, damping of the HOM's generated by a drive beam can be observed via the RF emission from the manifolds as well as by the deflection of a witness beam.

\section{THE PEP II ACCELERATING CAVITIES}

The accelerating cavities for PEP II are, due to the high average power requirements and to the importance of HOM damping, one of the more challenging elements of the PEP II project. During the last year we have participated in most of the regular weekly meetings at SLAC devoted to their design and engineering. Two issues in 
which our contributions have been specific are referenced in [2] and [3] and discussed briefly below.

\section{(a) The RF coupling design}

Two methods of coupling the driving RF power into the accelerating cavity are being considered: (1) loop coupling plus a co-ax to waveguide junction together with a co-ax window, and (2) iris coupling with a quarter-wave waveguide transformer and a circular waveguide window. Both designs are required to provide adjustable coupling to accommodate future beam current upgrades, the former can do so via loop rotation, the latter by rnaking the transformer assembly interchangeable. Both also need to incorporate a damping stub to damp a particular HOM which has a node near the apertures of the main damping waveguides. Reference [2] discusses two aspects of this problem: (1) calculation of coupling constants for the loop using MAFIA in combination with the Kroll-Yu and Kroll-Lin methods and comparison with experiment, and (2) damping of the above mentioned HOM using time domain MAFIA calculations starting with a MAFIA/KL determined loading field (as discussed in section $V$ of our previous progress report).

(b) Window design for the iris-transformer-waveguide configuration [3]

Over the past two years the design of a self matched thin (i.e. short compared to wavelength) circular window mounted in a short section of circular waveguide has been developed. The window assembly is matched to rectangular waveguides (of different dimensions) on either side of the assembly by placement within the circular section and choice of window thickness. A large standing wave with electric field maximum within the window is inherent to self matched window design. Because of the large average power requirements of PEP II, this leads to a large heating load and a requirement for a prestressed mechanical mounting to deal with the resultant thermal stress. A substantial engineering effort is going into implementation of this approach, and it is not considered to be risk free.

We (with Cho $\mathrm{Ng}$ ) have shown that a traveling wave design can reduce the heat deposition by more than a factor seven, which is sufficient to allow conventional mounting in place of the above mentioned prestressed mounting. A systematic design procedure based upon a set of MAFIA calculations (with some assistance from formulas in Marcuvitz) has been developed, and a number of designs have been generated. To simplify the window development program, our designs have been based upon the self matched configuration, and the traveling wave behavior is achieved by providing a reflecting iris on each side of the window. The currently favored configuration involves a pair of thick double gap capacitors. Capacitative (in contrast to inductive) reflectors lead to broader band performance and a more compact assembly. The double gap design makes the heat deposition more uniform and closer to the walls and reduces longitudinal fields at the window. The iris's are made thick to reduce the electric field in the gaps. Further refinements in design will be made as the PEP II cavity program evolves. Two talks at SLAC (outside the PEP II cavity meetings) have been given on this work, and a PEP II working paper is planned.

\section{MODE FILTERS}

The 2.93" diameter power transport guide planned for the NLC RF system propagates very many modes. The tapers and mode transducers which appear at the ends of various sections are excellent reflectors for unwanted modes so that there is the 
opportunity for a forest of narrow resonances in them, which may degrade the performance. Some mitigation of this problem may be obtained by the use of a mode filter. We have been studying various radial gap configurations for some time. Because quantitative experimental investigation of the effect of such gaps on unwanted modes is difficult (due primarily to the absence of suitable known sources for the unwanted modes and the expense of developing them), much of the investigation is being done via two parallel theoretical efforts.

The first of these involves a new two dimensional time domain MAFIA module which allows one to impose an outgoing wave boundary condition at a radial boundary as well as ingoing and outgoing wave axial boundary conditions on all propagating modes of a given azimuthal mode number $\mathrm{m}$. A number of bugs were found in the application of the module which were eventually resolved with the help of Tom Weiland who, fortunately, has been recently in residence at SLAC.

The second involved the development of a novel mode matching program in which slot radiation is simulated by superposing solutions with magnetic and electric boundary conditions at the ends of the slots. An important simplification in its implementation depended upon the extension of $S$ matrix symmetry to the generalized $S$ matrix discussed in Appendix 1.

What is calculated in both methods is the $S$ matrix for each $\mathrm{m}$ value, reduced in the sense that its matrix elements refer only to the propagating modes in the circular waveguide. The power which travels down the radial gaps (thus providing the filtering effect) is seen by the reduced $S$ matrix as an internal loss. Because we have been dealing with many propagating modes, sometimes as many as five with the same $m$ value (which requires a 10 by 10 symmetric matrix for its description), the amount of data required for a full description (and generated by these computations) is quite large. To reduce the complexity of comparing the two methods and comparing different mode filter designs we have defined a "loss figure" as the fractional total power lost to the gaps for a given input mode and consider it to be a figure of merit. In these computations, the loss figure is computed as the difference between the input power and sum of the powers carried by all outgoing waves all divided by the input power. For very small loss figure the method is very sensitive to errors in the determination of the individual $S$ matrix elements and loses accuracy. For many purposes, the fact that the loss figure is small is the only information one needs. An alternative method for computing the loss factor would be to compute the power out of the gaps from the fields at the outer gap boundaries. This would be straight forward to implement in the mode matching method (MM) and could also be accomplished by a post processor added to the MAFIA module, but neither of these have been done.

Reference [4] contains more details of the methods and tables of loss factors for all modes which propagate in 2.93" and 1.865" diameter circular waveguide at 11.424 GHz. The gap width studied is 0.25 ", and one, two, four, and eight gap configurations are considered. Where both methods have been used, the agreement between them is excellent, except for very small absorption cases where neither method is believed to be accurate for reasons given in the previous paragraph. Generally speaking, substantial loss factors are found for most incident modes, the exception being TE_mn modes for $m$ equals one and $n$ greater than one (and to a lesser extent, higher $m$ values as well).

It may be seen in ref [4] that the MM results given are more numerous than the MAFIA ones. This reflects the fact that as presently implemented MM is the more convenient for exploring a series of design options, and we have results for much larger numbers of gaps than are reported in [4]. The simultaneous development of the two 
methods played an essential role, however, in finding bugs and errors and achieving reliability.

Since the completion of [4], we (with Roger Jones) have implemented another approach to characterizing mode filter performance using MM. We consider a configuration consisting of a single gap with a pair of reference planes equally spaced on each side of the gap, and require that the fields on one reference plane be equal to the fields on the other one when multiplied by a constant. The constant may be thought of as an eigenvalue, the expansion of the field in waveguide modes as an eigenfunction, and the over all approach as the eigenvalue method. The eigenvalues are found to occur in reciprocal pairs, corresponding to attenuating waves propagating in opposite directions. The method is obviously convenient for considering large numbers of equally spaced gaps and for studying the effect of gap spacing.

Experience with earlier versions of mode filters have strongly indicated that the problem of disposing of the power coupled through the slots requires attention. The problem is complicated by the need to have a bakeable vacuum system and the need to control costs. Sami Tantawi has suggested the use of high loss magnetic steel for the gap walls. By using narrow gaps it may be possible to adequately attenuate the gap wave in a reasonable distance. With this in mind we have begun to apply the eigenvalue method to a system of many closely spaced narrow gaps.

In conclusion we should note that it has not been established that the use of mode filters will improve the efficiency of power transmission, but it is our view that this question can be addressed most effectively by studying the effect of mode filters whose electrical properties are known.

\section{THE PERSISTENT WAKEFIELD AND RELATED PROBLEMS}

This section refers to work carried out with Xin-Tian (Eddie) Lin and represents work which is providing the basis for his $\mathrm{PhD}$ dissertation. He has recently presented a report on the progress made in the past year to his UCSD research progress committee, and we are including this report as an appendix to the main text. We present a brief account of the main points here.

The persistent wakefield refers to that part of the wakefield which falls off as an inverse power of the time rather than exponentially. It is persistent in the sense that in a system without conductivity losses it eventually dominates any exponentially decaying wakefield. It has been discussed extensively in our previous reports. There are three new items.

(1) The waveguide cross cavity (see appendix) previously discussed with MAFIA time domain calculations has been reanalysed using a mode matching method. The method is novel in the sense that it succeeds in applying the technique to orthogonal boundaries rather than the usual parallel boundaries. The agreement with the earlier MAFIA work is good and MM is believed to give the more reliable result. The accuracy of the MAFIA method is limited by the need to numerically separate many overlapping effects. While the method has been applied only to the simplest case it is extendable by the well developed $S$ matrix cascading methods to more realistic cavity shapes and coupling irises. The orthogonal boundary MM has many other applications and as an illustration it has been used to calculate the $S$ matrix of $H$ plane and $E$ plane ninety degree junctions in rectangular waveguide. Excellent agreement is found with the Waveguide Handbook results. The method could provide an alternate approach to the mode filter problem discussed in the previous section. 
(2) An equivalent circuit approach to the study of enhancement of the persistent wake by the approach of the frequency of a damped resonance to the cutoff frequency. It is found that reducing the $Q$ of the resonance enhances the persistent wake, so that at a fixed distance from a source bunch and fixed damped mode frequency there is an optimum $Q$ which minimizes the wakefield. Overdamping increases the wakefield. The generality of the relation is under investigation.

(3) An analytic theory of multibunch transverse instability. The essential contributions of Marshall Rosenbluth, who became interested in the problem as a member of the research progress committee, are gratefully acknowledged. The method takes account of the cumulative field from all previous bunches and shows that for any decay rate greater than $1 / t$ there is always a particular bunch of a sufficiently long bunch train which experiences maximum deflection. The addition of bunches beyond that bunch does not increase the emittance growth. In connection with this general subject of emittance growth, we take this occasion to acknowledge a bug in the program used to estimate the growth reported in our previous progress report leading to a significant overestimate of the effect (see footnote 36 of [1]).

\section{PHOTONIC BAND GAP CAVITIES}

The work of the past year has been devoted to the study of metallic Photonic Band Gap (PBG) cavities and the results obtained are reported in ref [5]. The primary reason for this has been the fact that it is less expensive and less difficult to carry out an experimental program with such metal structures. Many issues, such as input, output, and inter-cavity coupling can be explored more easily with them, and the results should be directly applicable to the dielectric case. It is still our opinion that our long term interest should be in the dielectric case.

The computational methods which were used for our dielectric studies are not suitable for the metal PBG case, and we have replaced them with the finite difference methods which are traditionally used for analysing microwave cavities. There is one important respect in which the metallic case is simpler than the dielectric case, namely that it can be treated as a cylindrical structure with homogeneous cross section. Thus the spectrum obtained for the infinite length case of either polarization can be treated as the cutoff frequency spectrum for the TE and TM modes of a metallic waveguide, and the finite length case can be treated as a finite length waveguide cavity. In contrast with the dielectric case there is no TE-TM hybridization. There is also an infinitely degenerate set of TEM modes which have zero cutoff frequency and hence propagate at velocity $c$ in the axial direction. Modes of this type do not couple to an axial beam and will not be considered further. Apart from the TEM modes there is a large band gap at low frequencies which is the analog of the low frequency region of non propagation in metal waveguides.

As usual, a defect state is introduced by removing one metal cylinder. A monopole TM state is found, and a plot of the field distribution shows an energy density decay of $30 \mathrm{~dB}$ per lattice gap. This is much higher than that observed in the dielectric case and could be a potential advantage. The mode has also been observed in an experimental cavity built to our specifications by our SBIR partner AccSys. The test unit consisted of a $5 \times 5$ lattice of copper tubes brazed into copper plates separated by 0.8 " gap with other dimensions given in [5]. The defect mode was observed at $3.3 \mathrm{GHz}$. This corresponds to a TM_110 mode in typical waveguide cavity notation. Its TM_111 counterpart exists within the frequency range covered and was also observed. 
One of the issues which has been of concern to us has been the possibility of resonances within a region of band gap propagation. These are analogous to the waveguide damped resonances familiar for waveguide damped accelerator cavities of the sort discussed in [1]. These have been observed for the AccSys structure. Their observed $\mathrm{Q}$ is in the 100-200 range in contrast to the 4000 observed for the trapped monopole state. A possible mitigating feature of the band gap structure is the likelihood that the $Q$ of such modes depends upon their precise location within the propagation band. The possibility of shifting such modes into a region of better propagation by manipulating the geometry of the defect section is discussed in [5].

Plans for the future include adapting the structure for coupling in high power and carrying out experiments using power sources and shielding available at an appropriate test facility.

\section{OTHER PROJECTS}

I continue to attend the weekly meetings on pulse compression and power transport. The mode filter work reported in section III is of course related to that activity. SLED II is now operating with the circular waveguide $3 \mathrm{~dB}$ coupler replaced by a rectangular magic $\mathrm{T}$ with four flower petal mode transducers. It has been used to power accelerator test sections, both NLCTA related structures and a CERN structure.

I have interacted with R. Palmer, David Yu, and Shahar ben Menahim on the Cluster Klystron SBIR proposal and in particular on the design of power combiners, couplers, and mode transducers. Currently I have been advising Shahar on the design of the coax-waveguide junction for the klystron output including the choke assembly intended to block the propagating TE_11 mode in the coax section. This mode is generated by the junction, and it is desirable to prevent its propagating back to the klystron and distorting the cavity field.

My graduate student Xin-Tian (Eddie) Lin has been participating in a number of projects at SLAC with the object of broadening his experience and contributing to the effort. Thus he has worked with Dennis Palmer and Roger Miller on the RF gun, with K. $\mathrm{Ko}, \mathrm{C}$. $\mathrm{Ng}$, and S. Heifetz on impedence calculations for various components of the B factory rings, and with F. Bulos and G. Stupakov on the beam induced heating of a thin metal coating on a ceramic vacuum chamber.

\section{CONTRACT PUBLICATIONS AND REPORTS}

[1] K.A. Thompson,.N.M. Kroll, X.T. Lin., et al, Design and Simulation of Accelerating Structures for Future Linear Colliders, SLAC-PUB-6032 Nov. 1993, To appear in Particle Accelerators.

[2] K. Ko, C. Ng, N. Kroll, and R. Rimmer, Coupling Network Simulation for the PEP II RF Cavity, SLAC-PUB-6565 June 1994, To appear in the Proceedings of the 4 'th European Particle Accelerator Conference, London, UK, June 1994.

[3] M. Neubauer,.N. Kroll, et al, High Power RF Window Design for the PEP II B Factory, SLAC-PUB-6553 June 1994, To appear in the Proceedings of the 4 'th European Particle Accelerator Conference, London, UK, June 1994. 
[4] N. Kroll, K. Ko, and R. Jones, Mode Filter Design for the NLC Overmoded Power Transport System, SLAC-PUB-6623 Aug. 1994, To appear in the Proceedings of the 4'th European Particle Accelerator Conference, London, UK, June 1994.

[5] D.R. Smith, N. Kroll, and S. Schultz, Studies of a Metal Photonic Band Gap Cavity, To appear in the Proceedings of the Advanced Accelerator Concepts Workshop, Fontana, Wisconsin, June 1994

[6] N, Kroll, K. Thompson, K. Bane, R. Gluckstern, K. Ko, R. Miller, and R. Ruth, Higher Order Mode Damping for a Detuned Structure, SLAC-PUB-6616 Aug. 1994, To Appear in the Proceedings of LINAC94, Tsukuba, Japan, August 1994.

\section{DISCLAIMER}

This report was prepared as an account of work sponsored by an agency of the United States Uner any agency thereof, nor any Government. Neither the United States Government nor any agency theres liability or responsiemployees, makes any warranty, express or implied, or assumes any legal liability or responst, or bility for the accuracy, completeness, or usefulness of any information, apparatus, product, or process disclosed, or represents that its use would not infringe privately owned rights ence herein to any specific commercial product, process, or service by traty endorsement, recommanufacturer, or otherwise does not necessarily constitute or imply agency thereof. The views mendation, or favoring by the United States Government or any agency the and opinions of authors expressed herein do not 


\title{
Research Progress Report 94
}

\author{
Xintian Lin
}

July 31,1994

\section{Abstract}

Our previous work has demonstrated that the persistent wake field decays as $t^{-3 / 2}$ in waveguide damped cavity. We have also discussed its effect on Beam Break-Up instability.

This report will focus on our recent work in four aspects:

- We have implemented a new mode matching method to calculate the persistent wake of the crossed waveguide junction configuration. It involves mode matching on two surfaces perpendicular to each other.

- We have also derived the conditions satisfied by a general S-matrix involving non-propagating modes from complex Poynting's theorem.

- Using a circuit model, we have shown the interesting effect of iris on damped wake field and persistent wake field, and the minimum wake field achievable by waveguide damped cavity.

- Based on the solution of the transverse coupled beam equations[1], we have explained the effect of wake field decreasing on $\mathrm{BBU}$.

\section{Mode Matching}

Mode matching is a semi-analytical way to solve electromagnetic field in a structure. It involves expanding the field in two or more uniform regions in their respective eigenmodes and match them on the common surfaces. An illustrative example is a waveguide step, where waveguide changes its cross section at a certain place called interface. Fields are expanded on the left and right of the interface in their respective eigenmodes. Fields continuities force two infinite sets of equations involving unknown 
amplitude, one for the transverse magnetic field, and the other for the transverse electric field. Equations are then carefully truncated to yield a converging numerical result. [2]

We have implemented mode matching method on two perpendicular surfaces to calculate wake field and S-matrix.

\subsection{Wake Field}

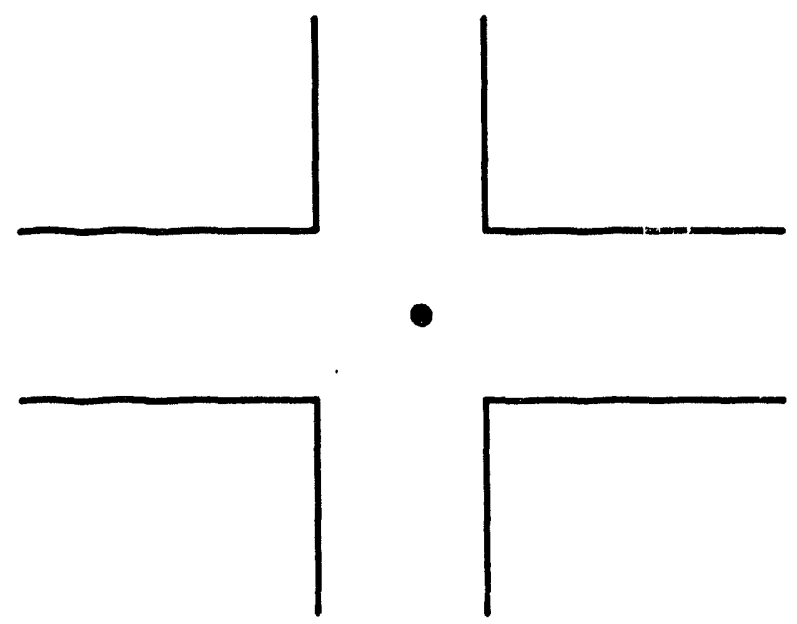

Figure 1: Crossed waveguides

The structure we are interested in is a crossed waveguide, see Fig. 1. Taking symmetry into consideration, the waveguide cross can be completely characterized by a 90 degree rectangular waveguide bend, see Fig. 2, with 3 possible boundary conditions on the outer bending boundaries: (Magnetic, Magnetic) for accelerating mode; (Magnetic, Electric) for dipole mode; (Electric, Electric) for quadrapole mode. Had the dimension of the waveguides been different, (Electric, Magnetic) would be for the other dipole polarization.

This waveguide junction is completely characterized by admittance matrix, which relates the port currents with port voltages. Current and voltage are defined from transverse fields by the following expression respectively:

$$
\begin{aligned}
& \vec{E}_{\perp,}=\sum_{n} e_{n} \vec{f}_{n}(x, y) \\
& \vec{H}_{\perp}=\sum_{n} i_{n} \vec{g}_{n}(x, y)
\end{aligned}
$$


where $\int \vec{f}_{n} \cdot \vec{f}_{m} d x d y=\delta_{n m}, \int \vec{g}_{n} \cdot \vec{g}_{m} d x d y=\delta_{n m}, \int \vec{f}_{n} \times \vec{g}_{m}^{*} \cdot d \vec{S}=-\delta_{n m}, d \vec{S}$ points outward. $e_{n}$ and $i_{n}$ are conveniently called voltage and current, and index $n$ runs through both surface and mode number of each terminal.

By specifying voltage $e_{q}$ on the surface, the magnetic field, which gives current $i_{p}$, can be calculated. Admittance matrix of this two-port surface at the waveguide junction is the linear coefficients relating $i_{n}$ 's with $e_{n}$ 's.

$$
i_{p}=\sum_{q} Y_{p q} e_{q}
$$

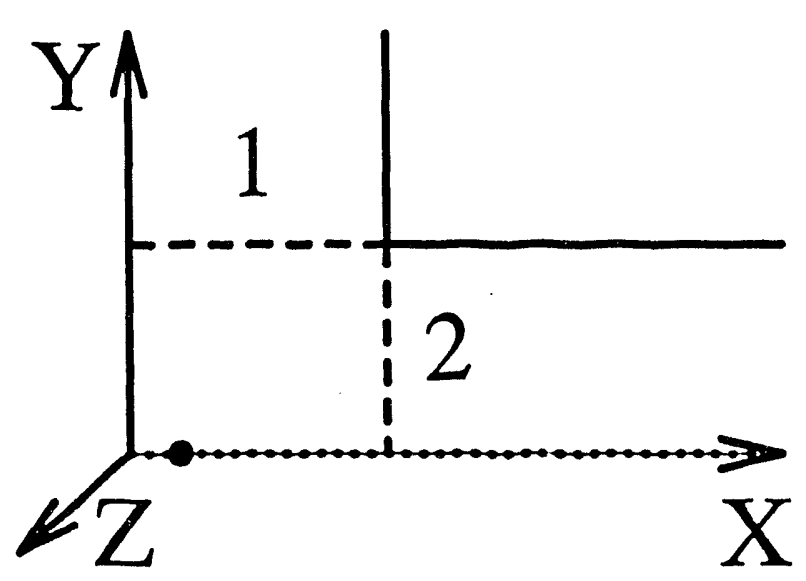

Figure 2: Short dashed line is the symmetric boundary, long dashed lines are the waveguide junctions, and the black dot is the beam position.

Beam excites wake field in the cavity. In circuit model language, beam produces current $\overrightarrow{i_{b}}$ on the terminal surfaces. By matching the field with outgoing wave condition, the resulting voltage on the surface can be easily solved:

$$
\begin{aligned}
& \vec{i}=\overrightarrow{i_{b}}+Y \vec{e}=Y^{c} \vec{e} \\
& \Rightarrow \vec{e}=\left(Y^{c}-Y\right)^{-1} \overrightarrow{i_{b}}
\end{aligned}
$$

where $Y^{c}$ is the diagonal matrix with the characteristic admittance of the outgoing wave. Vector notation is used here instead of indexes.

As a result, we can calculate dipole wake field $W_{\perp}=\mu_{0} H_{y}(\vec{e}) c$, where $\mathrm{c}$ is the speed of light.

The resulting persistent wake field is extracted from $W_{\perp}[3]$ :

$$
W_{p}(t)=\sum_{i} a_{i} t^{-3 / 2} \sin \left(\omega_{i}^{c} t+\phi_{i}\right)
$$

Compare amplitudes and phases with that from MAFIA calculation: 


\begin{tabular}{||c|c|c|c|c||}
\hline \hline & \multicolumn{2}{|c|}{ MAFIA } & \multicolumn{2}{c||}{ Mode matching } \\
\cline { 2 - 5 } & amplitude & phase & amplitude & phase \\
\hline$T E_{10}$ & $1.69539 \times 10^{7}$ & -0.8606519 & $1.73054 \times 10^{7}$ & -0.825266 \\
\hline$T E_{20}$ & $0.32437 \times 10^{7}$ & 0.6779432 & $0.32468 \times 10^{7}$ & 0.637734 \\
\hline$T E_{30}$ & $0.16199 \times 10^{7}$ & 0.8999303 & $0.157393 \times 10^{7}$ & 0.858344 \\
\hline \hline
\end{tabular}

They are in quite good agreement except a small phase discrepancy, which can be attributed to the numerical error of MAFIA. We calculated the persistent wake of a straighi waveguide shorted at one end, and found out that MAFIA result has to be corrected by a phase drift because it can't resolve the waveguide dimension accurately.

\subsection{S-matrix}

Voltage and current are not the only useful description of the terminal field, anoijer useful representation can be obtained from voltage amplitude or current amplitude as defined below:

$$
\begin{array}{rll}
e_{q} & =a_{q}+b_{q} \quad, \quad i_{q}=Y_{q}^{c}\left(a_{q}-b_{q}\right) \\
\text { or } \quad i_{q} & =a_{q}+b_{q} \quad, \quad e_{q}=Z_{q}^{c}\left(a_{q}-b_{q}\right)
\end{array}
$$

In Eq.(6) $a_{q}$ is interpreted as incoming wave voltage amplitude, $b_{q}$ as that of outgoing wave. $Y_{q}^{c}$ is the characteristic admittance of $q$ th mode. Eq.(7) is the dual of Eq.(6), where $a_{q}$ and $b_{q}$ are that of the current amplitude, and $Z_{q}^{c}$ is the characteristic impedance of the $q$ th mode.

Scattering matrix as defined by.

$$
b_{q}=S_{q p} a_{p}
$$

is readily derived from Eqs. (6) and (3):

$$
Y \vec{e}=Y(\vec{a}+\vec{b})=\vec{i}=Y^{c}(\vec{a}-\vec{b})
$$

Solve $\vec{b}$ in terms of $\vec{a}$ :

$$
\vec{b}=\left(Y^{c}+Y\right)^{-1}\left(Y^{c}-Y\right) \vec{a}
$$

Thus

$$
S=\left(Y^{c}+Y\right)^{-1}\left(Y^{c}-Y\right)
$$

This Matrix is naturally called voltage scattering matrix because it relates outgoing voltage amplitude with that of incoming. 


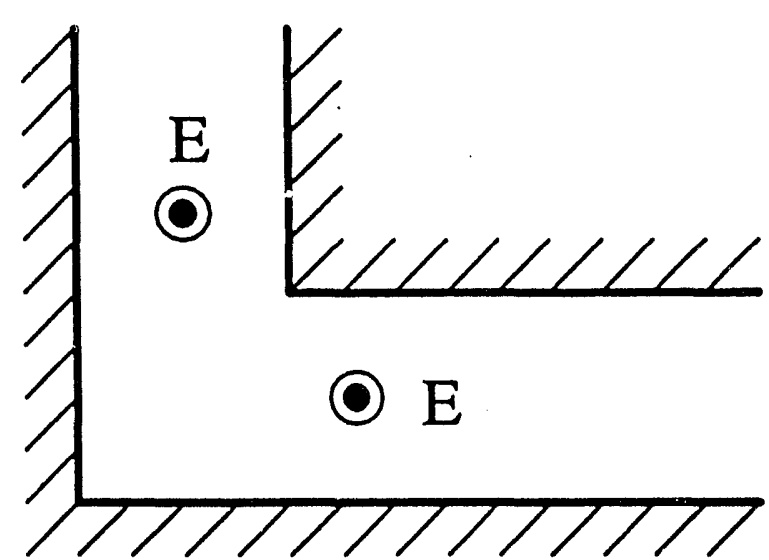

(a)

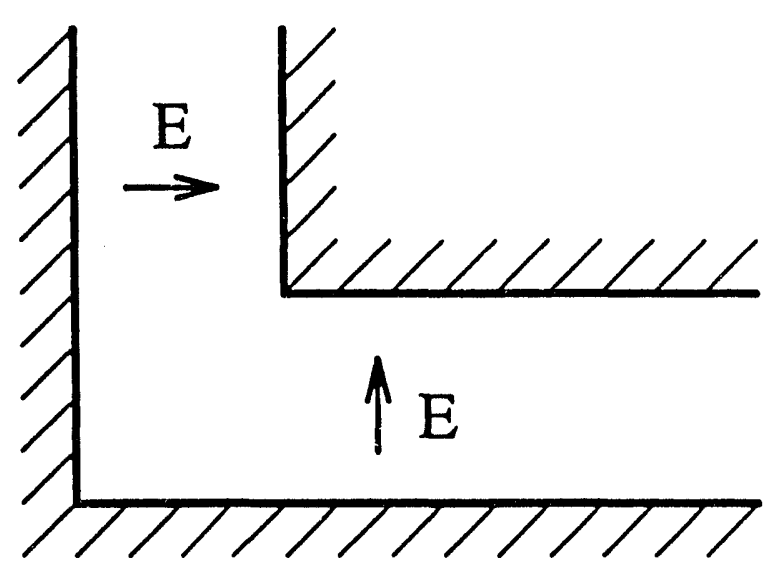

(b)

Figure 3: (a): rectangular waveguide bend with $T E^{01}$ mode, i.e. electric field pointing in/out of paper, called H-bend. (b) rectangular waveguide bend with $T E^{10}$ mode, i.e. electric field going across waveguide, called E-bend.

We calculated the S-matrix of a rectangular bend with only one propagating mode, see Fig. 3. The mode matching involves about 10 modes in each waveguide. The resulting amplitude and phase agree very well with that in the "Waveguide handbook".

\begin{tabular}{||c|c|c||}
\hline \hline$S_{11}$ & Handbook & Mode matching \\
\hline H-bend & $0.768831+0.0521746 \mathrm{I}$ & $0.770399+0.0526149 \mathrm{I}$ \\
\hline E-bend & $0.610224-0.30693 \mathrm{I}$ & $0.607852-0.30662 \mathrm{I}$ \\
\hline
\end{tabular}

Mode matching method works well with simple geometry. A little complicated regular shapes can also be dealt with by cascading S-matrix calculated by mode matching.

For example, a larger cavity attached to 2 waveguide can be decomposed into an asymmetry bend plus a step, shown in Fig. 4.

$S^{12}$ and $S^{23}$ can be calculated by mode matching. Normally, cascading can be handled by transfer S-matrix to T-matrix, multiply T-matrixes to get the total $\mathrm{T}$ matrix and then convert back to S-matrix. Because we used different number of modes in region 1 and 2,2 and 3, some matrix inversion is not well defined, we proceeded to write down s-matrix connecting incoming wave with outgoing wave, and eliminate the wave in region 2.

$$
\begin{aligned}
& a_{\text {out }}^{1}=S_{11}^{12} a_{\text {in }}^{1}+S_{12}^{12}\left(1-S_{22}^{23} S_{22}^{12}\right)^{-1}\left(S_{22}^{23} e^{-j k_{2} L} S_{21}^{12} a_{i n}^{1}+S_{23}^{23} a_{\text {in }}^{3}\right) \\
& a_{\text {out }}^{3}=S_{32}^{23} e^{j k_{2} L}\left(1-S_{22}^{12} S_{22}^{23}\right)^{-1}\left(S_{21}^{12} a_{\text {in }}^{1}+S_{22}^{12} e^{j k_{2} L} S_{23}^{23} a_{\text {in }}^{3}\right)+S_{33}^{23} a_{\text {in }}^{3}
\end{aligned}
$$

where the subscripts of S-matrixes refer to matrix elements related to corresponding regions. "in" and "out" refer to incoming and outgoing wave, respectively. 


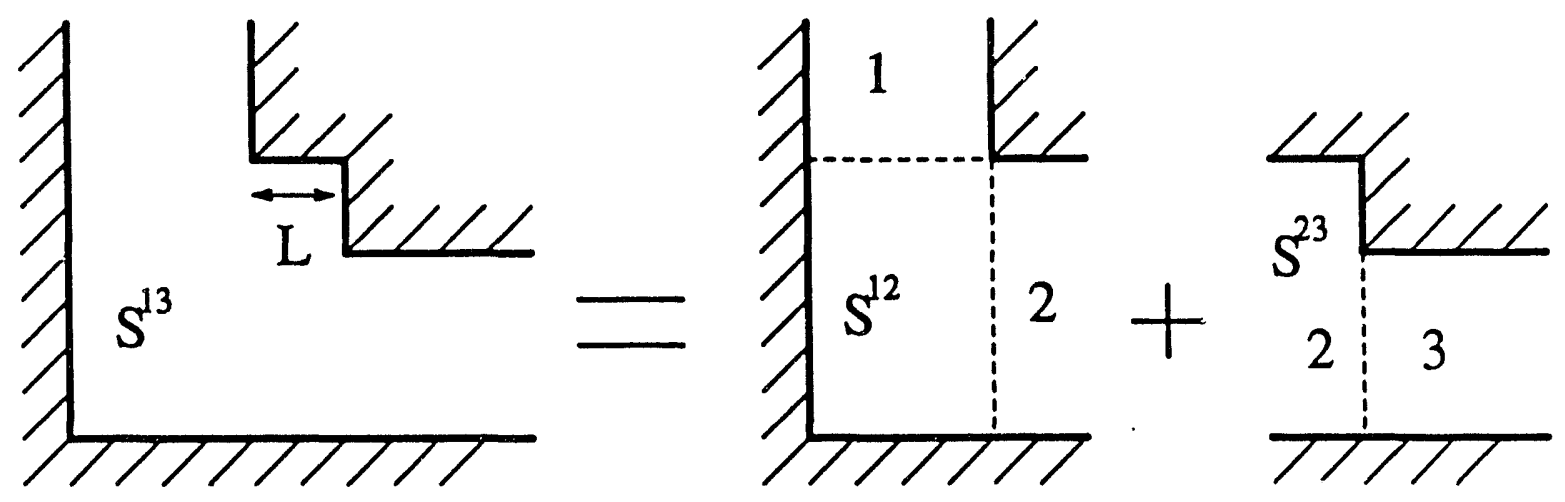

Figure 4: dashed line is the mode matching surface

The same method can be used to deal with iris and other regular shape elements. It can be extended to cylindrical coordinate and 3 dimensions as well.

\section{Conditions Satisfied by S-matrix Including Non Propagating Modes}

In numerical calculation, non-propagating modes are frequently encountered. Like in the cascading formula above, evanescent modes do penetrate the short microwave component. While widely used amplitude scattering matrix defined later in the section doesn't have an unitary condition when non-propagating modes involved because it can not be applied directly to Poynting's Theorem, we will show in this section that voltage scattering matrix does have such kind of condition.

\subsection{General Property of Waveguide Junction}

It's very difficult in general to calculate Electromagnetic field in a waveguide junction due to the complicated geometry involved. It has been pointed out [4] that a complete solution of an electromagnetic problem is not always desired. Often a description of conditions at the terminals of a junction is sufficient.

Consider a junction completely surrounded by an imaginary surface $S$. This surface cuts the waveguides perpendicularly at some planes called terminals.

Start from Poynting's Theorem:

$$
\int_{\imath}\left(\vec{E} \times \vec{H}^{*}\right) \cdot d \vec{S}=j \dot{\omega} \int_{v}^{\prime}\left(\epsilon|\vec{E}|^{2}-\mu|\vec{H}|^{2}\right) d V-\int_{v} \vec{E} \cdot \vec{J}^{*} d V
$$

where the surface integral only involves terminal, and the $d \vec{S}$ points outward. Expand 
the transverse field as in Eqs.(1, 2), Eq.(14) becomes

$$
\begin{aligned}
\sum_{n} e_{n} i_{n}^{*} & =\int_{v} \vec{E} \cdot \vec{J}^{*} d V-j \omega \int_{v}\left(\epsilon|\vec{E}|^{2}-\mu|\vec{H}|^{2}\right) d V \\
& =2 P+4 j \omega\left(W_{H}-W_{E}\right)
\end{aligned}
$$

$W_{H}$ and $W_{E}$ are the average magnetic and electric energy, respectively. A factor of 2 comes from time averaging. Eq.(15) provides a connection between the terminal quantities and the field quantities.

\subsection{Voltage Scattering Matrix}

The matrix $\mathrm{S}$ has several general properties of importance. One of them is its symmetry, arising directly from the symmetry condition of $Y$ and $Y^{c} . Y_{c}$ is diagonal, of course symmetric. Symmetry of $Y$ can be derived from Maxwell's equations.

$$
\begin{aligned}
& S^{T} Y^{c}=\left(Y^{c}-Y\right)\left(Y^{c}+Y\right)^{-1} Y^{c} \\
= & Y^{c}\left(Y^{c}+Y\right)^{-1} Y^{c}-Y\left(Y^{c}+Y\right)^{-1} Y^{c} \\
= & 2 Y^{c}\left(Y^{c}+Y\right)^{-1} Y^{c}-\left(Y+Y^{c}\right)\left(Y^{c}+Y\right)^{-1} Y^{c} \\
= & 2 Y^{c}\left(Y^{c}+Y\right)^{-1} Y^{c}-Y^{c} \\
= & Y^{c}\left[2\left(Y^{c}+Y\right)^{-1} Y^{c}-\left(Y^{c}+Y\right)^{-1}\left(Y^{c}+Y\right)\right] \\
= & Y^{c}\left(Y^{c}+Y\right)^{-1}\left(Y^{c}-Y\right)=Y^{c} S
\end{aligned}
$$

symmetry condition of $Y$ and $Y^{c}$ has been used in the first line.

Some additional conditions are imposed by Eq.(15). If Eqs.(6) and (8) are substituted in Eq.(15), there follows

$$
\begin{aligned}
\left(\vec{a}^{*}+\vec{b}^{*}\right) Y^{c}(\vec{a}-\vec{b}) & =\vec{a}^{*}\left(Y^{c}+S^{\dagger} Y^{c}-Y^{c} S-S^{\dagger} Y^{c} S\right) \vec{a} \\
& =2 P+4 j \omega\left(W_{H}-W_{E}\right)
\end{aligned}
$$

For a lossless junction, $P=0$, the real part of 1.h.s. vanishes

$$
\begin{aligned}
Y^{c}+Y^{c \dagger}= & S^{\dagger}\left(Y^{c}+Y^{c \dagger}\right) S+ \\
& S^{\dagger}\left(Y^{c \dagger}-Y^{c}\right)+\left(Y^{c}-Y^{c \dagger}\right) S
\end{aligned}
$$

Eq.(18) is the Generalized Unitary Condition(GUC) for the VSM $S_{v}$, the current scattering matrix $S_{i}$ follows the same derivation.

$$
\begin{aligned}
S_{i}= & \left(Z^{c}+Z\right)^{-1}\left(Z^{c}-Z\right) \\
S_{i}^{T} Z^{c}= & Z^{c} S_{i} \\
Z^{c}+Z^{c \dagger}= & S_{i}^{\dagger}\left(Z^{c}+Z^{c \dagger}\right) S_{i}+ \\
& S_{i}^{\dagger}\left(Z^{c \dagger}-Z^{c}\right)+\left(Z^{c}-Z^{c \dagger}\right) S_{i}
\end{aligned}
$$




\subsection{Comparison Between Voltage and Amplitude Scattering Matrix}

In microwave network analysis, amplitude scattering matrix is widely used [4] [5]. It follows the same derivation except in Eqs.(1) and (2), $\vec{f}_{n}$ and $\vec{g}_{\mathfrak{n}}$ are normalized:

$$
\vec{f}_{n} \Rightarrow \frac{1}{\sqrt{Y_{n}^{c}}} \vec{f}_{n} \quad, \quad \vec{g}_{n} \Rightarrow \sqrt{Y_{n}^{c}} \vec{g}_{n}
$$

and Eq.(6) changed into

$$
e_{q}=a_{q}+b_{q}, i_{q}=\left(a_{q}-b_{q}\right)
$$

the corresponding amplitude scattering matrix is

$$
S_{a}=\left(I+Y_{a}\right)^{-1}\left(I-Y_{a}\right)
$$

where $I$ is the unit matrix. Note here the admittance matrix $Y_{a}$ is related to $Y$ by scaling

$$
Y_{a}=\frac{1}{\sqrt{Y^{c}}} Y \frac{1}{\sqrt{Y^{c}}}
$$

It follows from the above two Eqs.

$$
S_{a}=\sqrt{Y^{c}} S_{v} \frac{1}{\sqrt{Y^{c}}}
$$

from Eq.(24), the well known symmetry condition of $S_{a}$ follows directly.

$$
S_{a}^{T}=S_{a}
$$

Then it's not surprising to see the symmetry condition of VSM $S_{v}$ in the light of the previous two equations.

The major difference between using $S_{a}$ and $S_{v}$ lies in the applicability towards Poynting's Theorem. Because of the normalization (or scaling) by $\sqrt{Y^{c}}, e_{n} i_{n}^{*}$ retains the meaning of power flow only when $Y^{c}$ is real, and that rules out Dissipative and Non Propagating (DNP) modes. Without DNP modes

$$
\begin{aligned}
\left(\vec{a}+\vec{b}^{*}\right)(\vec{a}-\vec{b}), & =\vec{a}^{*}\left(I+S_{a}^{\dagger}-S_{a}-S_{a}^{\dagger} S_{a}\right) \vec{a} \\
& =2 P+4 j \omega\left(W_{H}-W_{E}\right)
\end{aligned}
$$

with $P=0$,

$$
S_{a}^{\dagger} S_{a}=I
$$


GUC, on the other hand, not only contains the same information about scattering matrix elements connecting propagating modes, but also provides extra information about scattering matrix elements related to DNP modes.

Suppose we have a lossless junction with $N$ number of propagating modes and $M$ number of non-propagating modes, the characteristic admittance $Y^{c}$ and VSM $S_{v}$ can be written as

$$
Y^{c}=\left(\begin{array}{cc}
Y_{1} & 0 \\
0 & j B_{2}
\end{array}\right), S_{v}=\left(\begin{array}{ll}
S_{11} & S_{12} \\
S_{21} & S_{22}
\end{array}\right)
$$

where $Y_{1}$ is a $N \times N$ diagonal matrix with the $\mathrm{N}$ characteristic admittance of the propagating modes; $j B_{2}$ is a $M \times M$ diagonal matrix, note that it is purely imaginary. $S_{v}$ is also split in the same way. Plug them into Eq.(18)

$$
\begin{aligned}
& \left(\begin{array}{cc}
Y_{1} & 0 \\
0 & 0
\end{array}\right) \\
= & \left(\begin{array}{cc}
S_{11}^{\dagger} & S_{21}^{\dagger} \\
S_{12}^{\dagger} & S_{22}^{\dagger}
\end{array}\right)\left(\begin{array}{cc}
Y_{1} & 0 \\
0 & 0
\end{array}\right)\left(\begin{array}{ll}
S_{11} & S_{12} \\
S_{21} & S_{22}
\end{array}\right) \\
& -\left(\begin{array}{cc}
S_{11}^{\dagger} & S_{21}^{\dagger} \\
S_{12}^{\dagger} & S_{22}^{\dagger}
\end{array}\right)\left(\begin{array}{cc}
0 & 0 \\
0 & j B_{2}
\end{array}\right) \\
& +\left(\begin{array}{cc}
0 & 0 \\
0 & j B_{2}
\end{array}\right)\left(\begin{array}{ll}
S_{11} & S_{12} \\
S_{21} & S_{22}
\end{array}\right)
\end{aligned}
$$

It breaks down into 4 equations

$$
\begin{aligned}
Y_{1} & =S_{11}^{\dagger} Y_{1} S_{11} \\
0 & =S_{11}^{\dagger} Y_{1} S_{12}-S_{21}^{\dagger} j B_{2} \\
0 & =S_{12}^{\dagger} Y_{1} S_{11}+j B_{2} S_{21} \\
0 & =S_{12}^{\dagger} Y_{1} S_{12}+j B_{2} S_{22}-S_{22}^{\dagger} j B_{2}
\end{aligned}
$$

Eq.(32) is the same as Eq.(29) if we scale it. Eqs.(33) and (34) are complex conjugate of each other. The last three equations can be further simplified if we take generalized symmetry condition into consideration. Substitute Eq.(30) into Eq.(16)

$$
\left(\begin{array}{cc}
S_{11}^{T} Y_{1} & S_{21}^{T} j B_{2} \\
S_{12}^{T} Y_{1} & S_{22}^{T} j B_{2}
\end{array}\right)=\left(\begin{array}{cc}
Y_{1} S_{11} & Y_{1} S_{12} \\
j B_{2} S_{21} & j B_{2} S_{22}
\end{array}\right)
$$

then Eqs.(33) and (35) become

$$
\left.\begin{array}{r}
S_{21} S_{11}^{*}=S_{21}^{*} \\
\text { or }^{\prime} S_{11}^{*} S_{12}=-S_{12}^{*}
\end{array}\right\}
$$

It relates $S_{21}, S_{12}$ and $S_{22}$ with their complex conjugate respectively, i.e. a generalized reality condition. Current scattering matrix has similar results. 


\subsection{Conclusion}

We have derived voltage/current scattering matrix including DNP modes from admittance/impedance matrix and their corresponding generalized symmetry, unitary condition. Using a simple example, we have demonstrated the extra information contained in them compared with traditional amplitude scattering matrix. Besides the extra information, GUC is exact no matter where you do the mode number truncation, which allows one to check S-matrix in numerical calculation.

\section{Effect Of Coupling Iris On The Wake Field}

Using a lumped circuit, we can model the effect of coupling iris at the interface of waveguide and cavity:

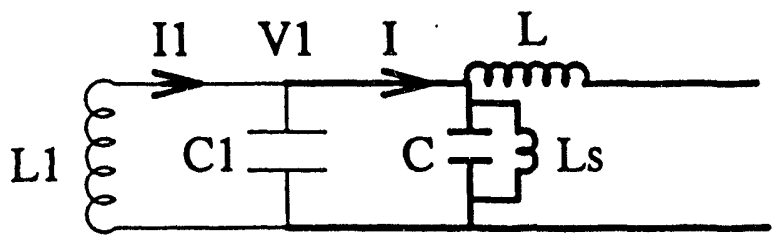

Figure 5: Thick line is a transmission line

It's a LC circuit loaded by a transmission line with shunt inductance to model the cut-off effect of waveguide Note that $L$ and $C$ are inductance and capacitance per unit length respectively, while $L$, is inductance times unit length. The capacitor is set up to have $V_{1}(t=0)=v_{0}$, and we will be watching the decay of the $V_{1}$

$$
\left.\begin{array}{rl}
\tilde{V}_{1} & =-L_{1} j \omega \tilde{I}_{1} \\
\tilde{I}_{1} & =-C_{1} v_{0}+j \omega C_{1} \tilde{V}_{1}+\tilde{I} \\
& =-C_{1} v_{0}+j \omega C_{1} \tilde{V}_{1}+\frac{\tilde{V}_{1}}{Z}
\end{array}\right\}
$$

where $Z=Z_{\infty} \frac{\omega}{\sqrt{\omega^{2}-\omega_{c}^{2}}}$ is the characteristic impedance of the transmission line, $Z_{\infty}=\sqrt{\frac{L}{C}}, \omega_{c}^{2}=\frac{1}{L_{c} C}$ is the cut-off frequency of the transmission line. Solve $\tilde{V}_{1}$

$$
\tilde{V}_{1}=\frac{-j \frac{\omega}{\omega_{0}} v_{0}}{\left(\frac{\omega}{\omega_{0}}\right)^{2}-j \frac{R}{z} \frac{\omega}{\omega_{0}}-1}
$$

where $\omega_{0}=\frac{1}{\sqrt{L_{1} C_{1}}}$, is the natural resonance frequency of the cavity, $R=\sqrt{\frac{L_{1}}{C_{1}}}$, is the characteristic impedance of the cavity. When $\frac{R}{Z\left(\omega_{0}\right)} \gg 1$ :

$$
\frac{V_{1}(t)}{v_{0}}=\frac{1}{2 \pi} \int \frac{\tilde{V}_{1}}{v_{0}} e^{j \omega t} d \omega
$$




$$
\begin{aligned}
& \approx \frac{1}{\sqrt{1-\frac{1}{4 Q^{2}}}} \sin \left(\sqrt{1-\frac{1}{4 Q^{2}}} t^{\prime}+\phi\right) e^{-\frac{t^{\prime}}{2 Q}} \\
& +4 \sqrt{\pi} \frac{-\frac{\omega_{c}}{\omega_{0}}}{\left(1-\left(\frac{\omega_{c}}{\omega_{0}}\right)^{2}\right)^{3 / 2}} \frac{1}{Q} \cos \left(\frac{\omega_{c}}{\omega_{0}} t^{\prime}+\frac{3}{4} \pi\right) \frac{1}{t^{3 / 2}}
\end{aligned}
$$

where $t^{\prime}=\omega_{0} t, Q=\frac{R}{Z\left(\omega_{0}\right)}, \phi=\pi-\sin ^{-1} \sqrt{1-\frac{1}{4 Q^{2}}}$.

It's very clear from the above expression that persistent wake amplitude is proportional to $\frac{1}{Q}$ which explains the phenomena we observed earlier. It also warrants our worry that as resonance frequency gets closer to waveguide cut-off, the persistent wake gets enhanced.

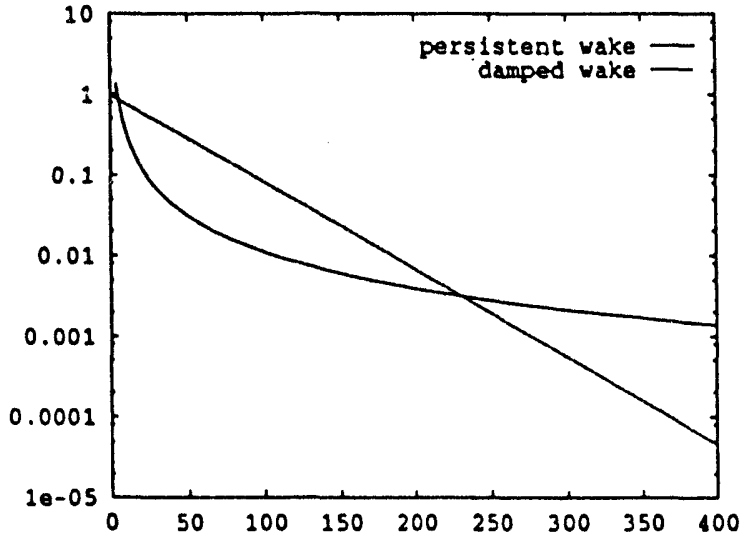

(a)

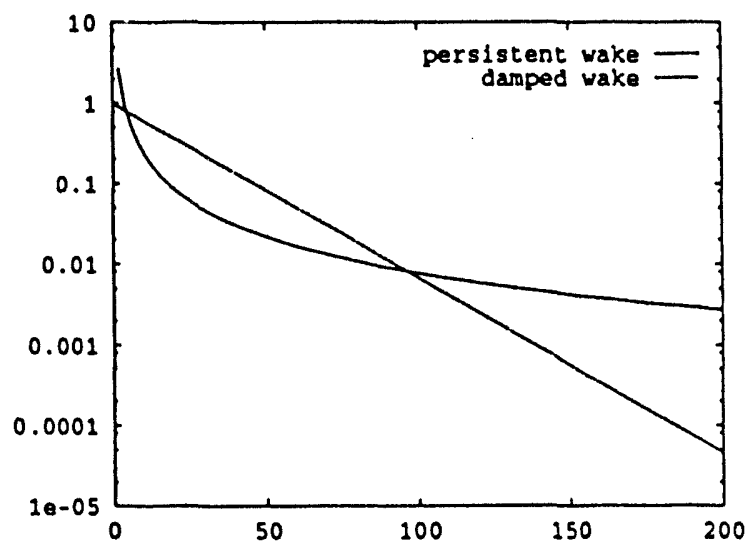

(b)

Figure 6: Comparison between the persistent wake and damped wake. (a): $Q=20$, $\frac{\omega_{c}}{\omega_{0}}=0.95$. (b):Q $=10, \frac{\omega_{c}}{\omega_{0}}=0.9$

From the two numerical examples, see Fig. 6, the effect of $Q$ is more prominent than that of the $\frac{\omega_{c}}{w_{0}}$. It seems unlikely that the latter effect will play a crucial role.

Eq.(41) also tells us the best waveguide damping can do at a certain distance $t^{\prime}$ behind the source particle. A typical value for NLC is $t^{\prime}=40 * \pi$, i.e. 20 wave length away. Take the envelop of Eq.(41)

$$
\begin{aligned}
W_{\perp} & =W_{0}\left(e^{-\frac{t^{\prime}}{2 Q}}+\frac{b}{Q} \frac{1}{t^{3 / 2}}\right) \\
b & =4 \sqrt{\pi} \frac{\frac{\omega_{c}}{\omega_{0}}}{\left(1-\left(\frac{\omega_{c}}{\omega_{0}}\right)^{2}\right)^{3 / 2}}
\end{aligned}
$$

Its minimum value occurred at

$$
\frac{1}{2 Q}=\frac{\frac{5}{2} \log t^{\prime}-\log b}{t^{\prime}}
$$


i.e. decreasing $Q$ beyond this value doesn't decrease the wake field at $t^{\prime}$. See Fig. 7

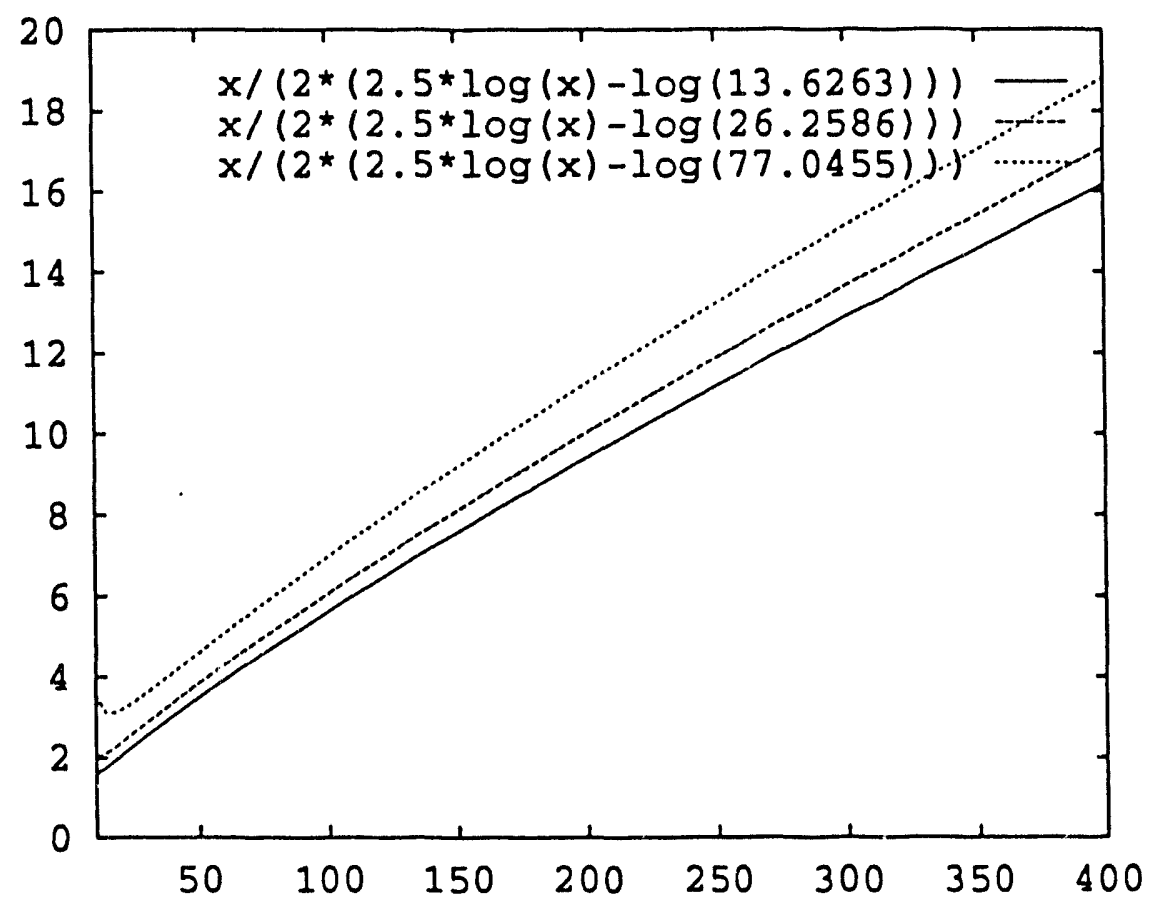

Figure 7: The three lines, from bottom to top, correspond to $\frac{\omega_{c}}{\omega_{0}}=0.7,0.8,0.9$, respectively. The horizontal axis is $t^{\prime}=\omega_{c} t$, and the vertical is the optimum $Q$ value

The minimum wake field that can be achieved at $t^{\prime}$

$$
W_{\perp}^{\min }=W_{0} t^{\prime-2.5}\left(5 b \log t^{\prime}+b-2 b \log b\right)
$$

Fig. 8 shows the limit of the damping effect of waveguide.

\section{Multibunch Transverse Instability}

In the linac of the proposed SLAC NLC design, $90 \mathrm{lnC}$ bunches are accelerated in a train. In fact, it's the de facto approach of all NLC design to increase luminosity. In this multi bunch design, one important problem that needs to be solved is the transverse Beam Break-Up (BBU) instability. A simple calculation points to linear growth of second bunch, quadratic growth of third bunch $\ldots t^{n-1}$ growth for the nth bunch, this more and more rapid growth for the later bunch poses a serious danger to emittance control. We will be addressing the problem of what type of wake field leads to stable bunch train for a given linac length. 


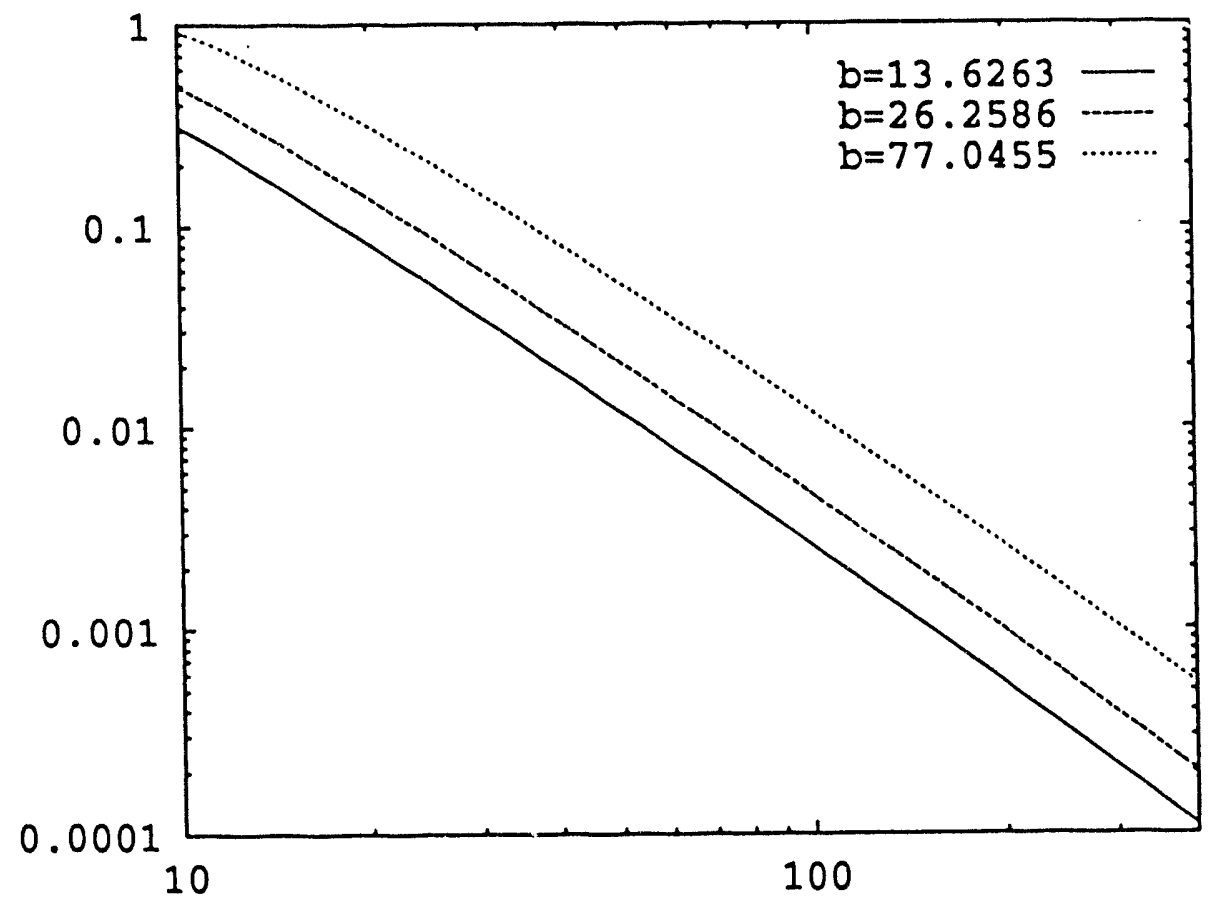

Figure 8: The three lines, from bottom to top, correspond to $\frac{\omega_{c}}{\omega_{0}}=0.7,0.8,0.9$, respectively. The vertical axis is the minimum wake field achieved as a ratio to the wake field at $t^{\prime}=0$ 
With proper scaling, this problem can be formulated by a series of recursive equations as follows: [1]

$$
x_{n}^{\prime \prime}+x_{n}=\lambda \sum_{m=1}^{n-1} x_{n-m} h(m)
$$

It assumes smooth focusing and no acceleration . $\lambda$ is a small coupling coefficient, $h(1)$ is conveniently chosen to be 1 . Take Laplace transform:

$$
-p \delta_{0, n}+\left(p^{2}+1\right) \tilde{x}_{n}(p)=\lambda \sum_{m=1}^{n-1} \tilde{x}_{n-m}(p) h(m)
$$

Without loss of generality, we assumed $x^{\prime}(0)=0$ and $x_{n}(0)=\delta_{0, n}$. Multiply Eq.(46) by $y^{n}$ and sum from 0 to $\infty$.

$$
\begin{aligned}
-p+\left(p^{2}+1\right) G(y, p) & =\lambda f(y) G(y, p) \\
\text { with } f(y) & =\sum_{m=1}^{\infty} y^{m} h(m) \\
G(y, p) & =\sum_{n=0}^{\infty} y^{m} \tilde{x}_{m}(p)
\end{aligned}
$$

We can solve the generating function $G$.

$$
G(y, p)=\frac{p}{p^{2}+1-\lambda f(y)}
$$

Inverse Laplace transform gives:

$$
G(y, t)=\cos (t \sqrt{1-\lambda f(y)})
$$

To find the behavior of $x_{n}$, we need to draw a contour in y plane around origin

$$
\begin{aligned}
x_{n}(t) & =\frac{1}{2 \pi i} \oint_{c} \frac{\cos (t \sqrt{1-\lambda f(y)})}{y^{n+1}} d y \\
& \sim \frac{1}{2 \pi i} \oint_{c} \frac{e^{i t \sqrt{1-\lambda f(y)}}}{y^{n+1}} d y
\end{aligned}
$$

Since our primary interest is in the behavior of $x_{n}(t)$ at large $t$, such as whether the beam blow up, how fast it grows; etc., we deform the contour to pass the saddle point for asymptotic evaluation, the saddle point satisfies:

$$
\frac{d}{d y} \phi(y)=\frac{d}{d y}(i t \sqrt{1-\lambda f(y)}-(n+1) \log y)=-\frac{i t \lambda f^{\prime}(y)}{2 \sqrt{1-\lambda f(y)}}-\frac{n+1}{y}=0
$$


The solution of Eq.(51) for each $n$ is substituted in $\phi(y)$ to get the exponential growth rate of $x_{n}$, and the maximum of those rates is the upper limit of BBU growth.

$$
\frac{d}{d n} \Re(i t \sqrt{1-\lambda f(y)}-(n+1) \log y)=0
$$

It is understood that the function is evaluated at the solt tion of Eq.(51).

$$
\frac{d y}{d n} \frac{d}{d y} \Re(i t \sqrt{1-\lambda f(y)}-(n+1) \log y)-\Re \log y=0
$$

the first term vanished at the saddle point, so

$$
y=e^{i \theta}
$$

$\theta$ can be calculated by invoking saddle point Eq.(51), assuming $\sqrt{1-\lambda f(y)} \approx 1$ :

$$
\Re\left(y f^{\prime}\right)=\sum_{m=1}^{\infty} m h(m) \cos (m \theta)=0
$$

the maximum growth rate is

$$
\gamma=\frac{\lambda t}{2} \Im f\left(e^{i \theta}\right)=\frac{\lambda t}{2} \sum_{m=1}^{\infty} \sin (m \theta) h(m)
$$

and it is occurred at bunch number $N_{\max }$

$$
N_{\max }=\frac{\lambda t}{2} \sum_{m=1}^{\infty} m h(m) \sin (m \theta)
$$

It's not surprising to see Eqs.(55)and (56). In fact, Eq.(55) is the maximum condicion of Eq.(56).

A few simple wake field models are worked out:

- $h(m)=\delta_{m, 1}, \theta=\frac{\pi}{2}, \gamma=\frac{\lambda t}{2}, N_{\max }=\frac{\lambda t}{2}$ (Daisy chain model)

- $h(m)=e^{-\beta(m-1)}, \cos \theta=\frac{1}{\cosh \beta}, \gamma=\frac{\lambda t}{2} \frac{1}{1-e^{-2 \beta}}, N_{\max }=\frac{\lambda t}{2} \frac{\tanh ^{2} \beta}{1-e^{-2 \beta}}$

- $h(m)=m^{-\frac{3}{2}}, \theta \approx 0.75, \gamma \approx 1.077 \frac{\lambda t}{2}, N_{\max } \approx 1.2899 \frac{\lambda t}{2}$

- $\lim _{c \rightarrow 0^{+}} h(m)=m^{-1+c}, \theta \rightarrow 0, \gamma \rightarrow \frac{\pi}{2} \frac{\lambda t}{2}, N_{\max } \rightarrow \infty \frac{\lambda t}{2}$ 
The same analysis allows one deal with oscillating wake field and all bunch initial offsets. No quantitative change.

It's interesting to note that all wake fields above produce a maximum beam growth rate, it has an interesting implication in accelerator design: If the wakefield decays faster than $\frac{1}{t}$, The luminosity can be indefinitely increased by adding more and more bunches to the train (we ignore the technical problems of beam loading etc.). In another word, multi bunch BBU is only a problem for the first few bunches.

Numerical simulations on different power law decaying field shown in Fig. (9) confirm the assertion.

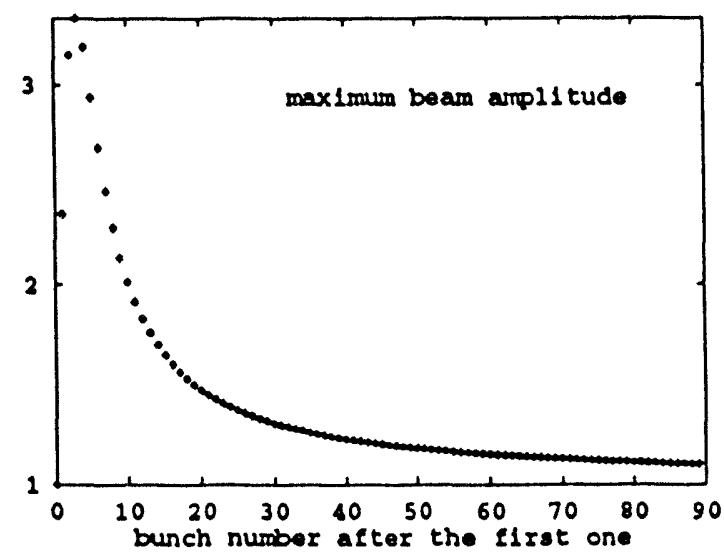

(a)

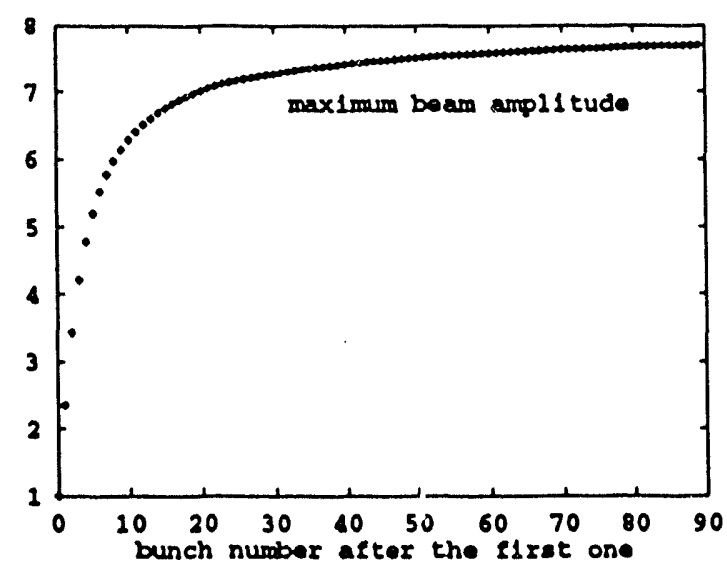

(c)

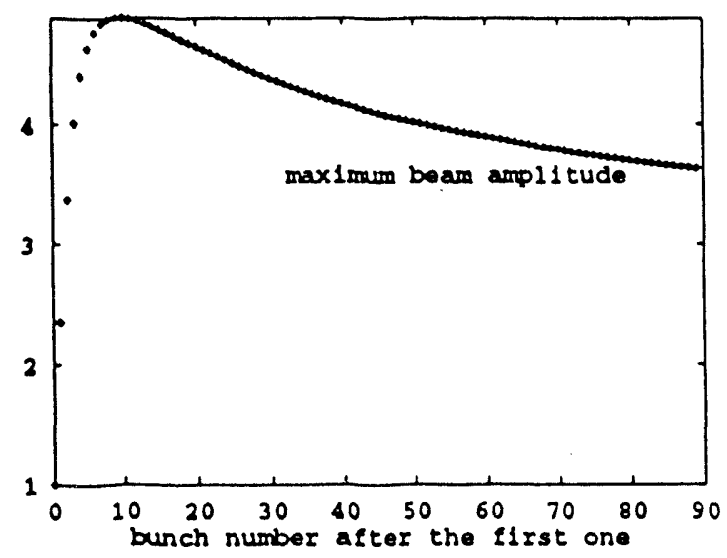

(b)

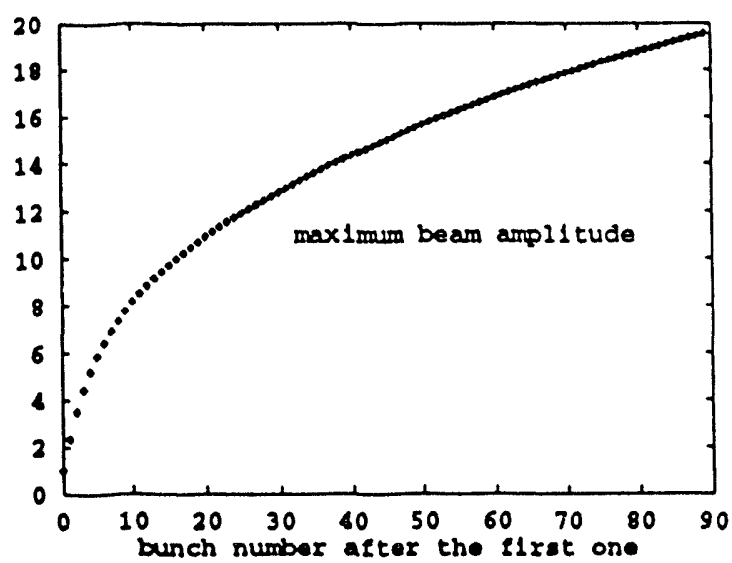

(d)

Figure 9: The trackings simulate the BBU under different wake field, all with $\frac{\lambda t}{2}=$ 2.145306, with initial offset $x_{n}(0)=1$. Maximum beam offset is plotted against bunch number. (a): $h(m)=m^{-1.5}$ (b): $h(m)=m^{-1.1}$ (c): $h(m)=m^{-1}$ (d) $h(m)=m^{-0.9}$ 


\section{References}

[1] Marshall Rosenbluth, private communication.

[2] R. Mittra, S. W. Lee Analytical Techniques in the Theory of Guided wave.

[3] Xintian Lin Research progress report 1993.

[4] C. G. Montgomery, R. H. Dicke, E. M. Purcell, Principles of Microwave Circuits. McGraw-Hill, 1948.

[5] R. E. Collin, Field Theory of Guided Waves. IEEE Press, 1991, pp 190-192 

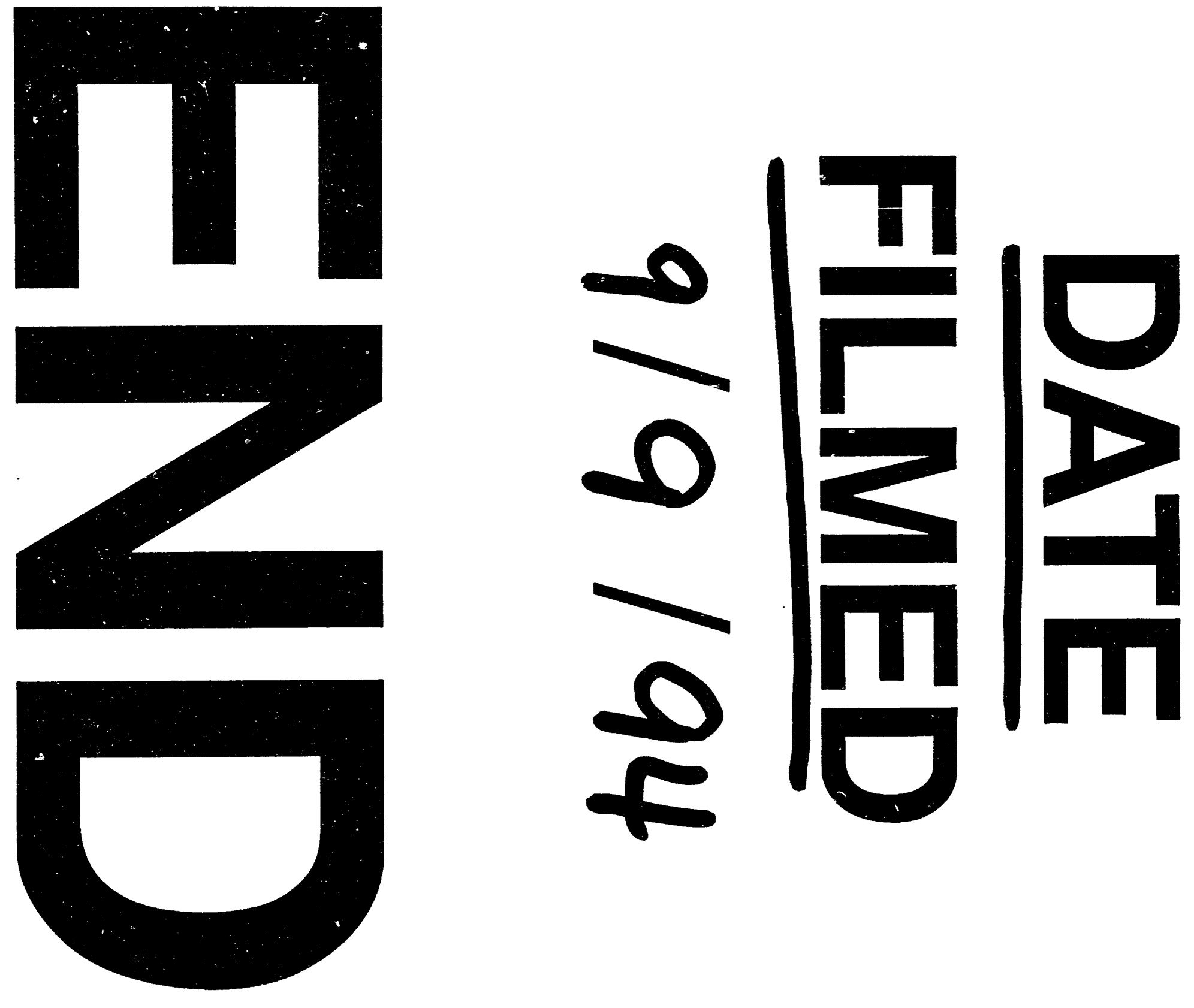
